\title{
From gas to satellitesimals: disk formation and evolution
}

\author{
Angioletta Coradini \\ INAF-IFSI \\ Via del Fosso del Cavaliere 100, 00133, Rome, Italy \\ E-mail address: angioletta.coradini@ifsi-roma.inaf.it
}

\section{Gianfranco Magni}

INAF-IASF

Via del Fosso del Cavaliere 100, 00133, Rome, Italy

E-mail address: gianfranco.magni@iasf-roma.inaf.it

\section{Diego Turrini}

INAF-IFSI

Via del Fosso del Cavaliere 100, 00133, Rome, Italy

E-mail address: diego.turrini@ifsi-roma.inaf.it

\section{Abstract}

The subject of satellite formation is strictly linked to the one of planetary formation. Giant planets strongly shape the evolution of the circum-planetary disks during their formation and thus, indirectly, influence the initial conditions for the processes governing satellite formation. In order to fully understand the present features of the satellite systems of the giant planets, we need to take into account their formation environments and histories and the role of the different physical parameters. In particular, the pressure and temperature profiles in the circum-planetary nebulae shaped their chemical gradients by allowing the condensation of ices and noble gases. These chemical gradients, in turn, set the composition of the satellitesimals, which represent the building blocks of the present regular satellites.

Keywords: Solar System: formation; Planets and Satellites: formation; accretion disks; Planets and Satellites: Jupiter; Planets and Satellites: Saturn 


\section{Introduction}

The satellite systems of Jupiter, Saturn and Uranus have been considered as miniature planetary systems since a long time (Greenberg, 1975). While they exhibit very diverse features, their study greatly helped to improve our understanding of the behavior of complex systems formed around a central body in terms of dynamical and physical properties. The regular satellites, which experience negligible solar perturbations, display dynamical properties similar to those of the planets. Moreover, the regular satellites are thought to have formed around their respective planets through a process similar to the one hypothesized for the accretion of the planets around the Sun. Any theory of the origins and evolution of the Solar System must then account for the presence and properties of satellite systems. When studying the origins of the satellites, we cannot avoid putting them in the context of the formation scenarios of their central planets. In fact, it is difficult to imagine satellite formation as totally independent from the environment generated and strongly affected by the central body. However, one has also to take into account that the formation times of the satellites are very short compared to those of their central bodies, and it is therefore possible for several generations of satellites to exist, each formed under different environmental conditions.

\section{From gas to satellitesimals}

If we examine the different satellite systems present in our Solar System we can find both regions characterized by negative outward density gradients and others which seem to have been dominated by stochastic collisional processes. The system of Jupiter exhibits a noticeable regularity. If we plot the so-called reconstructed mass of the satellites (i.e. the mass obtained by adding to each satellite the needed amount of volatile elements, in order to estimate the mass distribution in their formation regions) as a function of the distance from Jupiter (see Figure 1), the resulting density distribution is very regular. The Galilean satellites are a key example of a satellite system which co-formed with its parent planet, the forming satellites accumulating within a circum-planetary accretion disk during the final stages of the planetary formation process. The same doesn't apply to Saturn's system, which shows a large degree of variability (Greenberg, 1975). A large fraction of the mass in the Saturnian system resides in the largest satellite, Titan, which comprises more than $96 \%$ of the mass in orbit around the planet. Six other moons, prevailingly of icy composition, constitute roughly $4 \%$, of the total mass of the satellite system while the remaining 54 small moons, together with the ring system, only comprise about $0.01 \%$. The surface density computed through the reconstructed mass of the icy satellites (see Figure 1) shows alternating trends, both positive and negative. As a consequence, it is not straightforward to infer the characteristics of the original disk from the present features of the system like in the case of Jupiter. Moreover, in the Saturnian system stochastic phenomenon overlapped to the regular satellite formation process, namely an intense bombardment whose presence can be deduced from the overall structure of the icy bodies and from their surface crater distribution. 
Even more complex is the situation in the satellite system of Uranus, where regular satellites lying on the central plane of Uranus exist, thus orbiting the planet with an inclination of $98^{\circ}$ respect to the ecliptic planet. Thus, Uranus and its satellites form a pretty standard system while considered as an isolated dynamical system yet, once considered as part of the Solar System, they represent an anomalous property which must be accounted for by any theory of planetary formation and evolution. Following Greenberg (1975), one can make two hypotheses, depending on the time-scale of the change of the planetary obliquity. The first possibility is that, if the tilting time-scale was long (i.e. the change was gradual), the satellites could have undergone an adiabatic variation of their orbital angular momentum and could have survived the process. The second hypothesis assumes that the change was abrupt (e.g. due to a giant impact of a planetary embryo whose mass was $\sim 7 \%$ of that of Uranus, Safronov (1969)). If the tilting took place before the formation of the satellite system, it is plausible to assume that the circum-planetary disk disaggregated and reformed along the new equatorial plane and the satellites then formed in the new disk. If the tilting instead took place after the formation of a satellite system, the original system was probably destroyed and new bodies formed from the debris of the original ones. In this case the link between the satellite system and the formation of Uranus can be weak. Neptune represents another, different case: Goldreich et al. (1989) conjectured that Triton was captured from a heliocentric orbit as the result of a collision with one of Neptune's primordial regular satellites. The immediate postcapture orbit was highly eccentric, with its pericentric distance oscillating periodically. Dissipation due to tides raised by Neptune on Triton caused its orbit to evolve to its present state in $10^{9}$ years, with Triton being almost entirely molten for most of this time. While its orbit was evolving, Triton cannibalized most of the regular satellites of Neptune and also perturbed Nereid, thus accounting for the satellite's highly eccentric and inclined orbit. The only regular satellites of Neptune that survived were the ones which formed well within 5 Neptune's radii $\left(\mathrm{R}_{\mathrm{N}}\right.$ in the following) and they moved on inclined orbits as the result of chaotic perturbations forced by Triton. Goldreich et al. (1989) calculated that if Triton entered an elliptical orbit with a pericenter at $7 R_{N}$ and semimajor axis of $103 R_{N}$, then Triton could evolve, through tidal dissipation, to its present situation in less than one billion years. One consequence of the model proposed by Goldreich et al. (1989) was that Neptune would be devoid of satellites between $5 R_{N}$ and Triton's current orbit. When Voyager arrived three months later, this was found to be precisely the case. Also in this case the history of the present regular satellites is too complex to trace back the formation process.

Despite the differences previously described, Canup and Ward (2006) noted that the ratios of the overall masses of the regular satellite systems to those of their parent planets are about $10^{-4}$ for the systems of Jupiter, Saturn and Uranus. This suggests that a common process took place in all the satellite systems and in particular that the mass fraction of the satellite systems is regulated by the balance between the supply of inflowing material to the satellites and the satellite losses through orbital decay, driven by the interaction with the surrounding nebula. As a consequence, several satellite generations could have formed and got lost toward the central body, until the mass inflow onto the disk ended. The present satellites systems are likely the surviving members of the last satellite generation. However, in their work, the authors did not relate the mass accretion rate and the disk 
features to the formation time-scale and characteristics of the central body, so that it is almost impossible to date the formation time of the satellites.

Irregular satellites, instead, are believed to have formed in the Solar Nebula and to have been gravitationally trapped by the giant planet at a later time. Irregular satellites can supply information on the conditions of the surrounding space, as well as on the characteristics of the planets at the time when their trapping efficiencies were higher. The details and implications of the capture of the irregular satellites will be discussed later in the chapter. A complete and exhaustive model of satellite formation shall take into account of the conditions that were present in the Solar Nebula, that are the boundary conditions to be considered. However, connecting a model for the Solar Nebula to a model of giant planet formation that includes the formation of regular satellite systems is quite a difficult problem. Due to the strong link existing between the disk formation and the formation of the central body, we cannot avoid mentioning the current theories on the giant planets formation.

In the case of Jupiter and Saturn, the study of the formation conditions of the central bodies can be more directly linked to those of their surrounding disks, whose thermodynamic conditions, in turn, represent the boundary conditions for satellite formation and thus determine the initial chemistry of the satellites. Jupiter and Saturn are mostly gaseous and likely accreted a large part of their masses from the proto-Solar Nebula when the gas was still abundant. The state of the art on this subject has been reviewed by several authors (see e.g. Lunine et al., 2004; Lissauer \& Stevenson, 2007). The formation of giant planets is of paramount importance not only in order to understand our Solar System, but also to shed light on the possible nature and evolution of extraterrestrial planets. Moreover, following Guillot et al. (2004), we can state that Jupiter, owing to its large mass and rapid formation, played a crucial role in shaping the Solar System as we know it today. Jupiter mostly contains hydrogen and helium (more than $87 \%$ by mass) and as such it bears a close resemblance to the Sun. However, the Sun has only 2 $\%$ of its mass in heavy elements whereas Jupiter has between 3-13\%. The exact amount of these heavy elements in the planet and their distribution are key issues to understand how the Solar System formed (ibid), and the only way to correctly evaluate them is to know what the mass of the planetary core is. In fact, the idea that the atmospheres of the giant planets were similar in composition to the primordial nebula from which they formed has been largely disproved thanks to their study both from space and from Earth (Encrenaz 1994). Encrenaz (1994) notices that significant differences were measured in the abundances of helium, deuterium and carbon of the four giant planets, both between themselves and in respect to the Solar Nebula. In particular, the variations in the $C / H$ and $D / H$ ratios gave support to the "nucleation" formation scenario, in which the four giant planets first accreted a nucleus of about 10 Earth masses, big enough to gravitationally capture the surrounding gaseous nebula (ibid). One of the problems still open in this scenario is the mass of the central core that shall be considered. Goldreich, et al (2004) revised the problem of initial formation of planets and suggested that the region populated by the outer planets could have been characterized by the presence of many large bodies, of about the size of Uranus and Neptune, which could have formed even before the formation of inner planets, provided that the outer disk was richer in mass than the inner one. This means that the original disk from which the planets evolved contained the mass of 
a "minimum mass nebula" in the inner regions, but a much larger amount in the outer ones. Therefore, the core of Jupiter and Saturn could have formed at this time, much before the time when the gas dissipation in the nebula took place.

Another important consideration on the possibility to quickly form the central core of giant planets has been done by Kornet, Wolf \& Rozyczka (2007), who showed that the frequently adopted approximation that the surface density of the planetesimals is a constant fraction of the gas surface density cannot be accepted without care. In fact, these authors showed that the surface density of planetesimals can be locally enhanced by a large factor respect to the gas, thus speeding up the subsequent formation of the planets. This is due to the interaction between the gas and solid particles in the initial phases of evolution of the disk, as well as to the mutual interactions between the growing planetesimals. Therefore a detailed analysis of the initial conditions present in the Solar Nebula could help solving the problem of the formation of the cores of the giant planets.

\section{Gas capture and the formation of disks and planets}

The formation of Jupiter and Saturn can be modeled through the gas accretion onto a pre-existing solid core. This approach, which has been adopted by several authors, can be followed in all its different phases, provided that a detailed description of the gas accretion process and the correct evolution of the central body thermodynamics are considered. In fact, the central body evolution is not irrelevant for the thermal evolution of the disk surrounding it, and, in turn, the hydrodynamic evolution of the disk is responsible for the mass accretion rate onto the planet, which at this stage is the main energy source of the growing object.

For the rest of this section we will consider mainly the cases of Jupiter and Saturn, for which several authors make the following working hypotheses. First, a scenario in which the regular satellites form within circum-planetary accretion disks produced during the final stages of gas accretion (e.g. Lubow \& Ogilvie (2001); D'Angelo, Henning \& Kley (2002); Magni \& Coradini (2004)) is assumed. Second, for a given inflow rate of gas and solids, the circum-planetary gas disk is assumed to be in quasi-steady state due to the balance of the inflow supply and the disk's internal viscous evolution, assuming that the disk viscous spreading time is short compared to the timescale over which the inflow changes. Up to now, the existing hydrodynamic models generally used spherically symmetric proto-planets, consisting of a growing solid core and a solar composition gaseous envelope, surrounded by the accretion region. The accretion of Jovian planets, however, can be hardly assumed to be spherically symmetric, taking place in a strongly asymmetric region of several $\mathrm{AU}$, dominated by the gravity of the Sun and crossed by density waves. On the contrary, threedimensional models of the evolution of giant solar and extrasolar planets didn't include satisfactory physical description of the interfaces between the growing planet and the nebula (e.g. Artymowicz (1998); Rauer \& Hatzes (2007) and references therein). Lissauer et al. (2009) consider the gas accretion in a turbulent disk, whose evolution is computed in a three-dimensional space. This allows these authors to improve their modeling of Jupiter's formation, taking into account the time evolution of the gas flux throughout the Hill sphere: their modeling, however, is based on ad hoc assumptions on the gas capture criterion. Their 
results suggest accretion times for Jupiter ranging between several $10^{4}$ years to a few $10^{5}$ years, the accretion timescale strongly depending on the chosen physical parameterization and the disk turbulence. Moreover, while the influence of the gas accretion onto the planet is correctly taken into account, the feedback effects of the planetary formation on the disk are not explicitly considered (ibid).

The physical nature and the development of the core instability need to be discussed more deeply. First we have to introduce the concept of critical mass, i.e. the mass of the core beyond which the core starts collecting the surrounding gas through a hydrodynamic instability. Analytical arguments (Stevenson, 1982; Wuchterl, 1993) and numerical calculations (Mizuno, 1980) suggest that the onset of core instability occurs when the mass of the gaseous atmosphere around the core becomes comparable to the core mass itself. In a recent paper, Rafikov (2006) discusses the subject, determining the value of the critical mass in different conditions. This value depends on the physical characteristic of the atmosphere of the accreting object and mostly on the gas opacity. Rafikov (2006) systematically studied quasi-static atmospheres of accreting protoplanetary cores for different opacity behaviors and realistic planetesimal accretion rates in various parts of the protoplanetary nebula. In the region of the giant planets, he considered values of the critical mass as high as 20-60 Earth masses (for opacity values in the range $0.1-1 \mathrm{~cm}^{2} \mathrm{~g}^{-1}$ ) if planetesimal accretion is fast enough for protoplanetary cores to form prior to the nebular gas dissipation. If the core mass is much less than the critical value, the envelope/core mass ratio is very low, the self-gravitation of the gas is negligible and the maximum equilibrium radius of the envelope is much larger than the Hill radius. Therefore, there is a limit to the amount of gas that can be collected by the core from the surrounding nebula. When the core mass is near to the critical value, also the envelope radius is comparable to the Hill radius. For core masses greater than the critical value, a further increase produces a growth of the Hill's radius greater than the corresponding value of the envelope radius, and more and more gas can be collected from the nebula. At the same time, the heating due to the in-falling gas produces an expansion of the envelope up to the boundaries of the Hill's lobe. The gas accretion onto the core is thus driven by the thermodynamic and hydrodynamic conditions near the growing planet and, in turn, depends on the ratio between the energy carried by the in-falling gas and the energy associated with pressure effects. In addition, angular momentum is redistributed in the in-falling gas under the action of the gravitational fields of the Sun and of the growing planet. Moreover, fast radial hydro-dynamical instabilities can grow until they stop the accretion, producing a rebound of a significant fraction of the gas envelope out of the sphere of influence of the growing planet, as shown by Wuchterl (1991). Such effect, however, strongly depends on the assumed boundary conditions, particularly on the density and temperature of the gas in the Solar Nebula (Wuchterl, 1993) and on the adopted geometry. As a result of these combined effects, the accretion timescale is the maximum between the radiative timescale and the timescale needed by the external regions of the envelope to reach a hydrostatic quasi-equilibrium state. However, dissipative processes due to turbulence are present in the Solar Nebula and in particular around the growing planet. Here, angular momentum is dissipated inside the accretion disk formed by the in-falling gas producing a substantial flux of matter towards the core of the protoplanet, strongly shortening the accretion timescale. Thus, the final gaseous accretion of the central planet occurs via formation and replenishment of a disk-shaped envelope embedded in the Hill lobe, which 
suggests again that the rapid collapse of a spherical gaseous envelope is not a valid description of the process. A 3D hydrodynamics code must be used to trace the accretion. In the next section we'll describe our three dimensional model of planetary accretion in some detail to supply a template of the relevant processes and their treatment.

\section{Evolution of the circum-planetary disk: a template model}

In what follows we will describe the structure and the results of our model of circum-planetary disk evolution, in order to show what are the critical parameters affecting the satellite formation process. To do that, we have to set some preliminary definitions. We will call growing planet the central body in nearly hydrostatic equilibrium, containing a planetary core composed by a mixture of rocky and icy materials plus a spherical gaseous envelope. Surrounding the growing planet is a deeply anysotropical region where gas accretion is supersonic and turbulence is present: this region will be referred to as the accretion disk (Shakura \& Sunyaev, 1973). Outside the accretion disk is the Hill's lobe, the large spheroid region where gas accretion takes place and through which material captured from the Solar Nebula evolves inward. These three regions represent a useful schematization of the history of the gas which enters the sphere of influence of the growing planet. The accretion of the central body has been treated assuming an annular region, centered on the Sun, as the planet-feeding zone. The radial extension of the feeding zone should be large enough to include the region where the gravitational and dynamical effects (i.e. the density waves induced by the planet on the gas of the Solar Nebula) due to the growing planet are significant.

The reference system is rigidly rotating with an angular velocity equal to the Keplerian orbital velocity at the location of the planet. The numerical integration of the system of partial differential equations is performed by using a first order explicit numerical scheme, the so called donor cells method described by Boss (1980). An advantage of this method is that it ensures rigorous conservation of the global mass and momentum through the numerical grid. Moreover, in those regions of severe mass depletion, the method prevents a negative density by taking sufficiently small time steps. Note that tidal and Coriolis forces are present, since the grid is rotating with the growing planet. With this scheme we can properly account for the mass and angular momentum transfer from the Solar Nebula to the planet. However, due to the discrete structure of the hydrodynamics equations, angular momentum is no longer exactly conserved as it would be in an inertial, non rotating reference frame. The shorter is the timescale of the spurious diffusion, the more the angular momentum conservation is violated. The timescale of the spurious diffusion can be increased by increasing the mesh grid-points number, at the cost of increasing the computational time: how to achieve a balance between these two competing needs has to be evaluated depending on the aims of the simulations. The grid covers a shell around the present orbit of the planet which has more elements in the volume surrounding the protoplanet itself (i.e. accretion zone) than elsewhere around the orbit. The angular vertical thickness of the feeding zone is $10^{\circ}$, i.e. large enough to contain more than one 
pressure height-scale. Due to the reasons previously presented, the radial extension of the feeding zone has been assumed ranging from 2.7 AU to 13.7 AU.

The hydrodynamic evolution is driven by the ordinary mass, momentum, energy conservation and radiative diffusion equations. The external forces are the pressure forces, plus non-inertial forces, Coriolis and centrifugal, and gravity forces due to the Sun and to the growing planet. In addition, it is also present a first order treatment of gas self-gravitation that may be non-negligible inside the Hill's lobe and near the growing planet, due to the strong density enhancement. The self-gravity term is computed starting from the radially averaged density around the growing planet. The equations are expressed in spherical coordinates and in the Eulerian approach.

The effects of turbulent viscosity on the gas are directly treated into the hydrodynamic equations of momenta adding the ordinary nonlinear terms (Landau \& Lifschitz, 1971) like pseudo-acceleration terms. The main parameter involved in the turbulent terms is the so-called alpha parameter (Shakura and Sunyaev,1963) that tunes the strength of the turbulence itself. Turbulent dissipation causes a general outward transfer of angular momentum in the nebula and an inward flux of matter. In the region around the growing planet, the geometry of the forces causes respectively outward and inward fluxes of angular momentum and mass. So, turbulent viscosity causes an enhancement of the mass accretion rate of the growing planet. Since around the growing planet the adopted grid geometry contrasts with the natural planet-centered geometry of the fluxes, we considered averaged values of the radial fluxes. The value of alpha has been assumed to be 0.05 in the case of Jupiter, and 0.01 in the case of Saturn. This has been done since the examination of the structure of the Jovian disk exhibits a much higher degree of turbulence then the Saturnian one. The accretion timescale for Saturn has been found to be from two to four times that of Jupiter. In this calculation, the accretion timescales for Jupiter and Saturn results between a few $10^{3}$ and about $10^{5}$ years, depending on the value of the alpha parameter. In Figure 2 we plotted the density distribution on the mid-plane for the accretion regions around Jupiter and Saturn respectively. The two strongly anisotropic patterns show the regions where the accreting gas is concentrated before entering the envelope of the growing planet. The central region is again nearly isotropic due to the combined effects of the non inertial forces, of the pressure and of the viscosity, which cause the rotation to change from retrograde to prograde. So, in the region close to the planet a Keplerian, gravitationally bound prograde disk can be identified. The region from which the protoplanet collects the gas, i.e. the feeding zone, is shown in Figure 3. The feeding zones of Jupiter and Saturn don't overlap between themselves, thus allowing treating the formation of the two planets independently. The thermal evolution of the disks has been evaluated integrating the total energy equation and including the radiative time-dependent diffusive terms.

\section{The disk evolution and chemistry}

As already stated, the gas is captured either by the planet directly, or through the formation of an accretion disk, depending on the gas angular momentum distribution. The structure of the growing planet, and, in particular its mass, radius 
and luminosity, depends on the mass accretion rate. The distribution of the gas swirling around the planet depends, in turn, on the planet's thermodynamic characteristics: a fraction of the energy collected by the planet through mass capture is in fact released back to the disk as thermal energy. The region surrounding the growing planet is heated up, the pressure in the region increases, and a pressure barrier reduces the mass flow (Magni and Coradini, 2004). This is a negative feedback effect that tends to increase the planet growth timescales and affects the overall disk thermodynamics.

The energy production rate per unit mass is assumed to be constant throughout the planet and the energy acquired by the planet is virialized. Then the energy flux rate can be computed simply as the ratio of the total energy flux per unit mass that enters the region occupied by the planet. The opacity coefficient has been computed by combining the results of different authors: we refer the readers to Magni \& Coradini (2004) for details on the opacity treatment. The equation of state, linking the pressure to the other thermodynamic variables, includes both partial dissociation of $\mathrm{H}_{2}$, ionization of $\mathrm{H}$ and $\mathrm{He}$, as well as electronic degeneracy that can become important in the inner part of growing planet. The dimension, the structure and the global parameters of the circum-planetary accretion disk are evaluated starting from the angular momentum distribution, referred to the growing planet, of the cells inside the Hill's lobe.

Valuable models for Jupiter and Saturn acts to consider realistic cases: a minimum mass Solar Nebula (about 0.02 solar masses) from the initial critical value (15 Earth masses) up the final planetary mass in order to study accretion timescales, mass distribution and thermal evolution in the regions corresponding to that of the accretion proto-satellite disk can be used in order to investigate the environment in which regular satellites. The mass of the feeding zone has been artificially depleted after the planet reached its final mass in order to simulate the natural process of depletion due to the gap formation and/or the process of dissipation of the nebula driven by the solar wind. We have obtained a sequence of quasi-stationary models of the region occupied by the proto-satellite accretion disk characterized by decreasing masses and optical depths, and studied the saturation pressures of the main chemical volatile components, like $\mathrm{H}_{2} \mathrm{O}, \mathrm{CO}$ , $\mathrm{CO}_{2}, \mathrm{CH}_{4}, \mathrm{NH}_{3}, \mathrm{Ar}, \mathrm{Ne}$. In Figure 5 we show the temperature distribution in the disk of Jupiter and Saturn at the different phases of their accretion processes. As we previously said, those two disks are characterized by different values of the average alpha parameter: in the case of Jupiter (alpha=0.05), the disk is more turbulent then in the case of Saturn (alpha=0.01).

The final phases of accretion, described here, are slow: the accreted gas generates a disk that gradually cools down, allowing for the condensation of different materials. To follow these phases in a quantitative way, we have computed the evolution of the temperature in the disk. However, these calculations take into account the average variation of the temperature, since different parts of the disk can be characterized locally by different values of the thermodynamic quantities. In fact, the structure of the disk is very complex: the external region is strongly perturbed by the interactions with the gas of the Solar Nebula, while the internal regions are more dominated by the interaction with the protoplanet. 
The temperature of the Jovian disk keeps almost constant for a large fraction of its history in all the regions in which we have divided the disk for sake of simplicity. These regions correspond respectively to $0-40 R_{J}, 40-50 R_{J}, 60-80 R_{J}, 80-100 R_{J}$ and 100-150 $R_{J}$. Obviously, the inner region $\left(0-40 R_{J}\right.$, i.e. the one which is relevant for satellite formation) is the hottest one. Toward the end of the accretion, when the mass inflow reduces, the disk cools down. At this stage, the Jovian disk reaches about $125^{\circ} \mathrm{K}$ in the region between $0-40 \mathrm{R}_{\mathrm{J}}$, and $68^{\circ} \mathrm{K}$ in the region between 80-100 $\mathrm{R}_{\mathrm{J}}$. The Saturnian disk is subdivided in the same number of regions and is characterized by a similar behavior, but the disk is generally colder. At the end of the accretion phase, the temperature becomes quite uniform in the entire disk, stabilizing at about $39^{\circ} \mathrm{K}$.

The gas pressure in the disks can be computed also as a function of the accretion time: the resuls are shown in Figure 7. As the temperature, the global gas pressure and the partial pressures of the different gases decrease as the mass inflow slows down. The mean values of the ratios between the disk pressure and the saturation pressure of the different gases present in the disk are shown in the same Figure. When the ratio is greater than unity, the component is in gaseous form; when it is lower, the thermodynamic conditions in the disk allow for a phase transition from gas to solid. In Jupiter's case (Figure 7, panels a-c), only $\mathrm{H}_{2} \mathrm{O}$ condensation is possible in the formation region of the satellites $\left(0-40 R_{J}\right)$ and only in the final phases of the accretion. When the accretion stops, other species start to condense, such as $\mathrm{NH}_{3}$ and $\mathrm{CO}_{2}$ ices, both in the inner and outer regions. The emerging picture is very interesting since it hints that a certain amount of ammonia has been incorporated in the satellites. The presence of ammonia in the formation region of the satellites is important: ammonia, if incorporated in the ice or under the more complex molecule of a clathrate, can contribute to the thermal history of satellites since it strongly lower the freezing point of the material in which it's incorporated. This analysis implies that the Jovian disk was dominated since the beginning of the history of the disk by the presence of $\mathrm{H}_{2} \mathrm{O}$ grains. Silicate particles were also present in the Jovian disk. Icy particles contributed, with the silicate grains, to the formation of satellitesimals in the disk: the combination in different proportions of these two main components plays the most important role in the determination of the average density of the satellites.

In the case of Saturn the situation is different and the condensation of $\mathrm{CO}_{2}, \mathrm{NH}_{3}$ and $\mathrm{CH}_{4}$ grains is possible (see Figure 7, panels d-f). At this stage, the Saturnian disk is mostly cold, with only the internal regions having temperatures which allow the sublimation of different ices, thus permitting a complex chemistry to evolve. Highly volatile gases were incorporated in the mixture since the very beginning of the life of the disk. Even $A r$ can be incorporated in the satellitesimals in the external part of the disk, in agreement with the puzzling feature of the atmosphere of Titan, where no primordial noble gases other than argon were detected by the Gas Chromatograph Mass Spectrometer (GCMS) aboard the Huygens probe during its descent to Titan's surface on January 14, 2005. The $C O$ ice is not condensed in the Saturnian sub-nebula. As a consequence, the satellitesimals formed in the disk could be depleted of this ice as suggested by Mousis et al. (2008). This would explain the deficiency of $C O$ observed in Titan's atmosphere (Alibert \& Mousis, 2007). Instead, $\mathrm{H}_{2} \mathrm{O}, \mathrm{NH}_{3}, \mathrm{CO}_{2}$, and $\mathrm{CH}_{4}$ would remain incorporated in solids. The complex chemistry present in the Saturnian 
disk can thus be responsible for the internal evolution and differentiation of the satellites.

On the surfaces of the icy satellites, high volatility ices could be lost during the further evolutions of these bodies: the icy moons, in fact, were intensively bombarded and underwent to strong interactions with both solar and planetary radiations, as well as by high energy electrons and protons trapped in the radiation belts of Saturn. Only Titan has preserved large part of the original chemistry.

Water ice is abundant on Saturnian satellites, as shown by near-infrared spectra returned by VIMS (see chapter by Dalton et al., this book). The ice phase can be used to study the crystalline order of the surface ice on all the satellites. It could also be a way to measure the lattice order of ice that, in turn, depends on its condensation temperature and rate, its temperature history, and its radiation environment. Inner satellites are characterized by a larger content of crystalline ice, in respect to the amorphous one (that is almost absent), as measured on the basis of the strength of the absorption band at 2 and $2.05 \mu \mathrm{m}$.

The role of carbon compound was also important for the overall chemistry together with the water ice particles. Satellitesimals formed in the Jovian and the Saturnian disks would probably maintain records of the original composition. A similar analysis performed on the $\mathrm{CO}_{2}$ ice seen by VIMS shows that a large amount of "organic contaminants" was present in the Saturnian system. The radial distribution of the "contaminants" across the Saturnian system can be measured through the visible spectral slopes (ibid). The satellites have red (positive) slopes in this range, probably caused by the presence of a still unidentified UV organic absorber. Therefore, we can argue that the original chemistry is not totally cancelled by the overall evolution of the satellites.

It is at this point in the evolution of the disks that the model developed by Canup \& Ward (2002) becomes important. In fact, there is a final phase in their evolution where disks are in quasi-steady states and cold, as their model assumes. The previously presented results aren't in disagreement with Canup's and Ward's modeling, but on the contrary confirm their assumptions in a rigorous way. The further evolution of the disk could imply displacements of the satellitesimals as well as capture and bombardment from external bodies. These subjects will be discussed in the second part of this chapter (see Mosqueira, Estrada and Turrini, this book).

\section{Bibliography}

Alibert Y., Mousis O., "Formation of Titan in Saturn's subnebula: constraints from Huygens probe measurements", 2007, Astronomy and Astrophysics, 465, 1051-1060

Artymowicz P., "On the formation of eccentric superplanets" in "Brown Dwarfs and Extrasolar

Planets", 1998, Eds. R. Rebolo, E. L. Martin, M. R. Zapatero Osorio, Astronomical Society of the

Pacific, San Francisco, ASP Conference Series, 134, 152-161

Bodenheimer P., Grossman A. S., DeCampli W. M., Marcy G., Pollack J. B., "Calculations of the evolution of the giant planets", 1980, Icarus, 41, 293-308 
Boss A. P., "Protostellar formation in rotating interstellar clouds. I - Numerical methods and tests", 1980, The Astrophysical Journal, 236, 619-627?

Boss A. P., "Evolution of the SN. VI. Giant Gaseous Protoplanets Formation", 1998, The Astrophysical Journal, 503, 923-937

Cameron A. G. W., "Physics of the primitive solar accretion disk", 1998, Moon \& Planets, 18, $5-40$

Canup R. M., Ward W. R., "A common mass scaling for satellite systems of gaseous planets", 2006, Nature, 441, 834-839

Coradini A., Cerroni P., Magni G., Federico C., "Formation of the satellites of the outer solar system - Sources of their atmospheres" in "Origin and Evolution of Planetary and Satellite Atmospheres", 1989, Eds. S. K. Atreya, J. B. Pollack, M. S. Matthews, University of Arizona Press, Tucson, 723-762

D'Angelo G., Henning T., Kley W., "Nested-grid calculations of disk-planet interaction", 2002, Astronomy \& Astrophysics, 385, 647-670

Encrenaz T., "The chemical atmospheric composition of the giant planets", 1994, The Earth, Moon \& Planets, 67, 77-87

Greenberg R., "The Dynamics of Uranus Satellites", 1975, Icarus, 24, 325-332

Goldreich P., Murray N., Longaretti Y., Banfield D., "Neptune’s story", 1989, Science, 245, 500504

Goldreich P., Lithwick Y., Sari R., "Final stages of planet formation", 2004, The Astrophysical Journal, 614, 1024-1037

Guillot T., Stevenson D. J., Hubbard W. B., Saumon D., "The Interior of Jupiter" in "Jupiter. The planet, satellites and magnetosphere.", 2004, Eds. F. Bagenal, T. E. Dowling, W. B. McKinnon, Cambridge University Press, 35-57

Kornet K., Wolf S., Rozyczka M., "On the diversity of giant planets Simulating the evolution of solids in protoplanetary disks", 2007, Planetary \& Space Science, 55, 536-546

Landau L., Lifschitz E., "Mechanique des fluides", 1971, Mir Editions, Moskow

Lubow S. H., Ogilvie G. I., "Secular Interactions between Inclined Planets and a Gaseous Disk", 2001, The Astrophysical Journal, 560, 997-1009

Lunine J. I., Coradini A., Gautier D., Owen T. C., Wuchterl G., "The Origin of Jupiter" in "Jupiter. The planet, satellites and magnetosphere.", 2004, Eds. F. Bagenal, T. E. Dowling, W. B.

McKinnon, Cambridge University Press, 19-34

Lissauer J. J., "Formation of the giant planets", 1987, Icarus, 69, 249-265

Lissauer J. J., Stevenson D. J., "Formation of giant planets" in "Protostars \& Planets V", 2007,

Eds. B. Reipurth, D. Jewitt, K. Keil, University of Arizona Press, 591-606

Lissauer J. J., Hubickyj O., D'Angelo G., Bodenheimer P., "Models of Jupiter's growth incorporating thermal and hydrodynamic constraints", Icarus, 199, 338-350

Magni G., Coradini A., "Formation of Jupiter by nucleated instability", 2004, Planetary \& Space Science, 52, 343-360

Mayer L., Quinn T., Wadsley J., Stadel J., "Formation of Giant Planets by Fragmentation of Protoplanetary Disks", 2002, Science, 298, 1756-1759 
Mizuno H., "Formation of the Giant Planets", 1980, Progress of Theoretical Physics, 64, 544-557 Mousis O., Lunine J. I., Thomas C., Pasek M., Marbœuf U., Alibert Y., Ballenegger V., Cordier D., Ellinger Y., Pauzat F., Picaud S., "Clathration of Volatiles in the Solar Nebula and Implications for the Origin of Titan's Atmosphere", 2008, The Astrophysical Journal, 691, 17801786

Pollack J. B., Podolak M., Bodenheimer P., Christofferson B., "Planetesimal dissolution in the envelopes of the forming, giant planets", 1986, Icarus, 67, 409-443

Pollack J. B., Hubickyj O., Bodenheimer P., Lissauer J. J., Podolak M., Greenzweig Y., "Formation of the giant planets by concurrent accretion of solids and gas", 1996, Icarus, 124, 6285

Rafikov R. R., "Atmospheres of protoplanetary cores: critical mass for nucleated instability", 2006, The Astrophysical Journal, 648, 666-682

Rauer H., Hatzes A., "Extrasolar planets and planet formation", 2007, Planetary \& Space Science, 55,535

Safronov V. S., "Evolution of the protoplanetary cloud and formation of the Earth and planets", 1969, Nauka, Moscow, translation into english by the Israel Program for Scientific Translations, 1972, NASA TTF-677

Shakura N. I., Sunyaev R. A., "Black holes in binary systems. Observational appearance.", 1973, Astronomy \& Astrophysics, 24, 337-355

Stevenson D. J., "Formation of the giant planets", 1982, Planetary and Space Science, 30, 755-764

Wuchterl G., "Hydrodynamics of giant planet formation III: Saturn nucleated instability", 1991, Icarus, 91, 5364

Wuchterl G., "The critical mass for protoplanets revisited - Massive envelopes through convection", 1993, Icarus, 106, 323-338

\section{Figures}

\section{Figure 1}

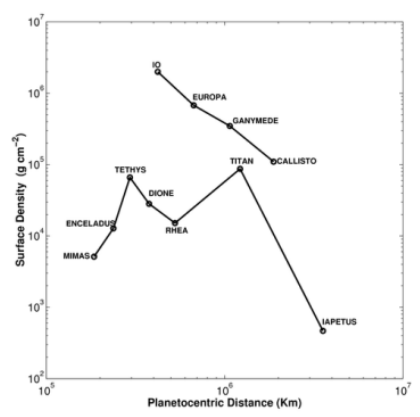

Figure 1: Surface density of the circum-planetary disks of Jupiter and Saturn estimated from the reconstructed masses of the satellite systems. The reconstructed mass is obtained by adding to each satellite the needed amount of volatile elements to achieve solar composition. Surface density is expressed in $\mathrm{g} \mathrm{cm}^{-2}$ while radial distance from the central planet is expressed in $\mathrm{Km}$. 


\section{Figure 2}
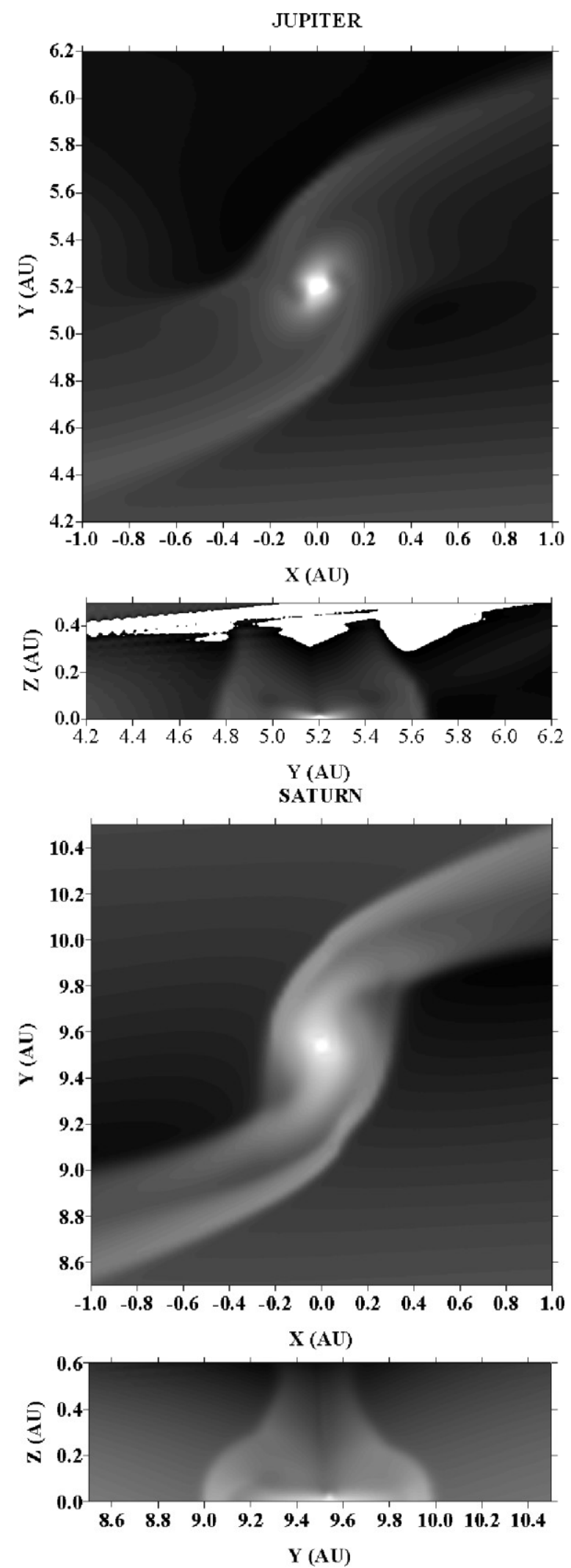

Figure 2: Density distributions in the accretion regions of the growing planets during the final stages of their formation. For each planet, the upper plot represents the mid-plane of the Solar Nebula while the lower plot shows the vertical section along the Sun-planet direction. All 
distances are expressed in astronomical units while density is expressed in $\mathrm{g} \mathrm{cm}^{-3}$ and in logarithmic scale.

\section{Figure 3}
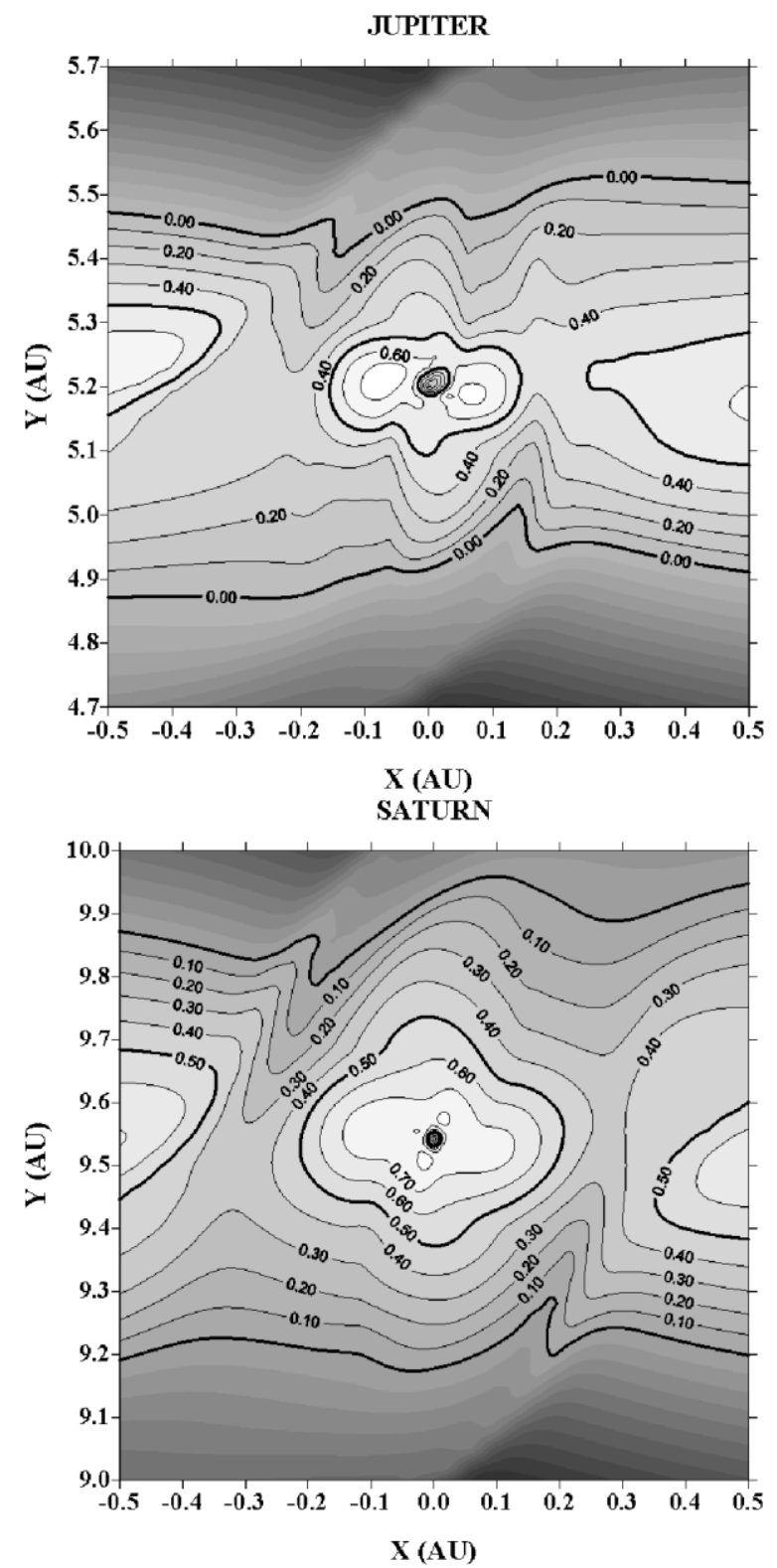

Figure 3: Mapping of the tangential velocities of the in-falling gas for Jupiter and Saturn at the same evolutionary stage as Figure 2. Tangential velocities are expressed in units of the local circular Keplerian velocity while distances are expressed in astronomical units. The iso-level curves refer to the regions of prograde motion around the planet. The Jovian Hill's radius is about $0.35 \mathrm{AU}$ while the Saturnian one is about $0.43 \mathrm{AU}$. 


\section{Figure 4}
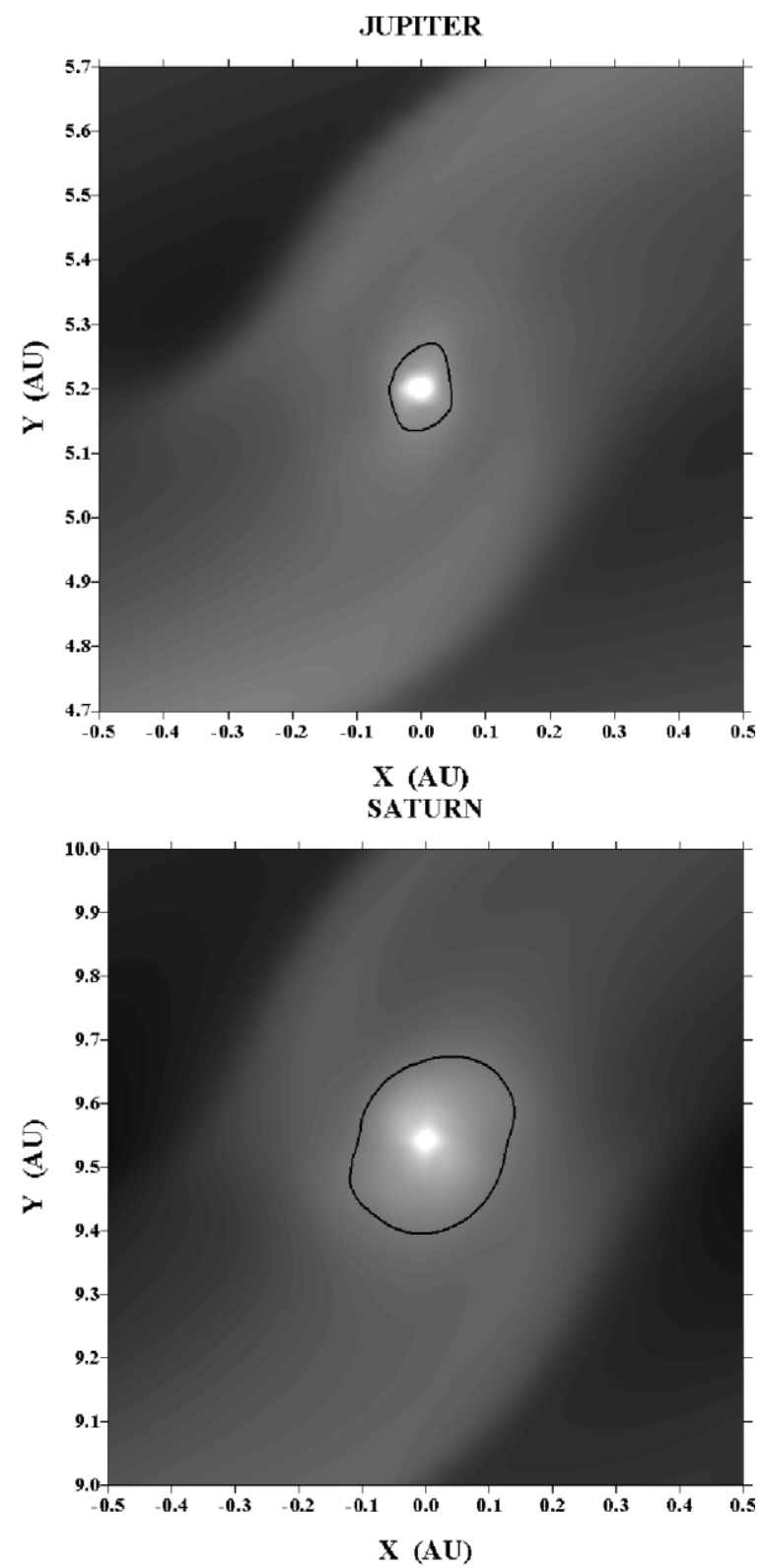

Figure 4: Surface density distribution for Jupiter and Saturn at the same evolutionary stage as

Figure 2. The black iso-curve refers to a density value of $1 / 30$ of the central density. The surface density is expressed in logarithmic scale in cgs units while distances are expressed in astronomical units. 
Figure 5
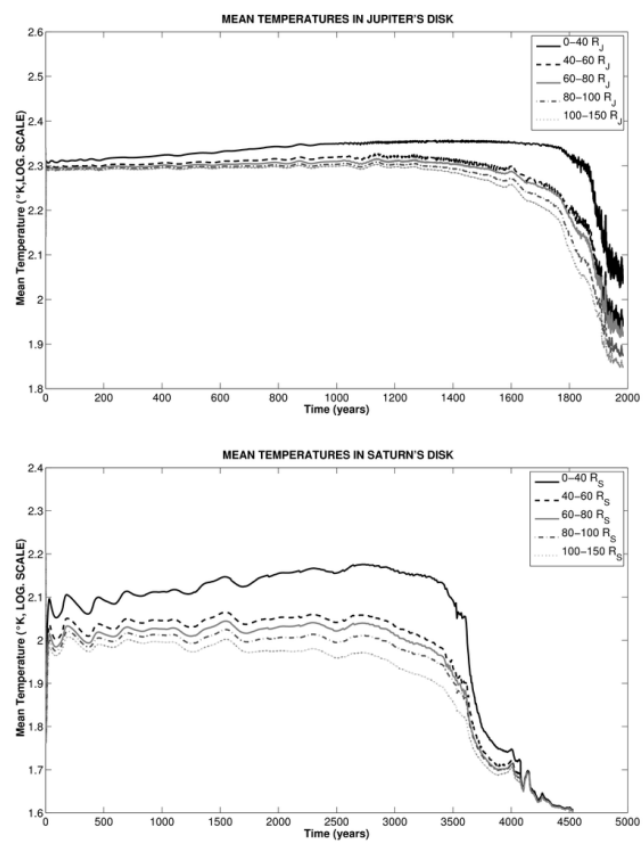

Figure 5: Mean temperatures in the Jovian and Saturnian disks during the formation of the respective planets. Each disk has been divided into five annular shells centered on the forming planets and the temperatures are averaged over each shell. Temperatures are expressed in ${ }^{\circ} \mathrm{K}$ and in logarithmic scale while the time is expressed in years.

\section{Figure 6}

\section{Jupiter}

\section{Panel a}

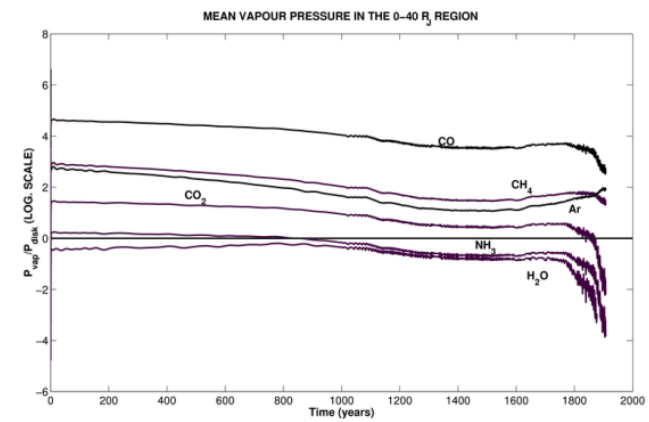

\section{Panel b}




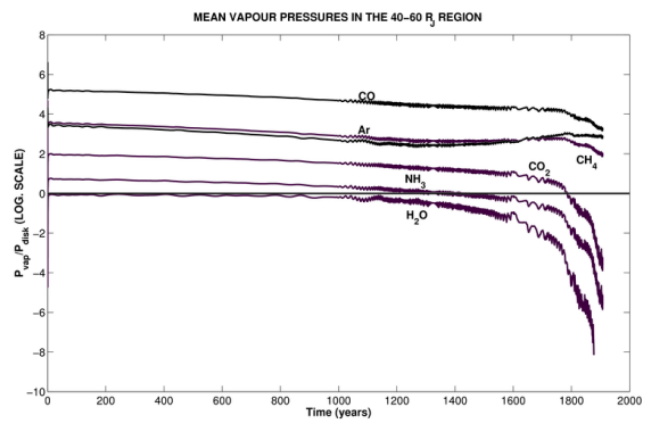

Panel c

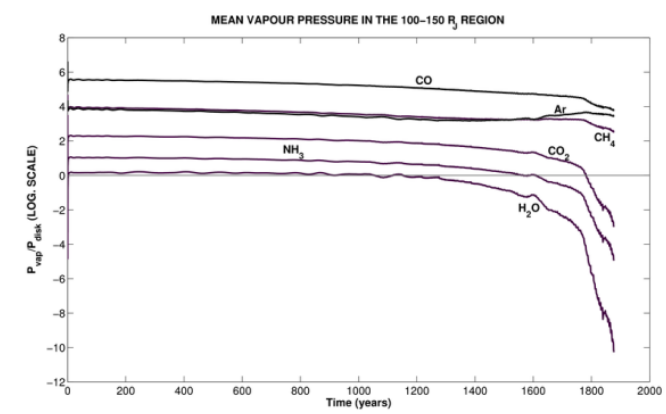

Saturn

\section{Panel d}

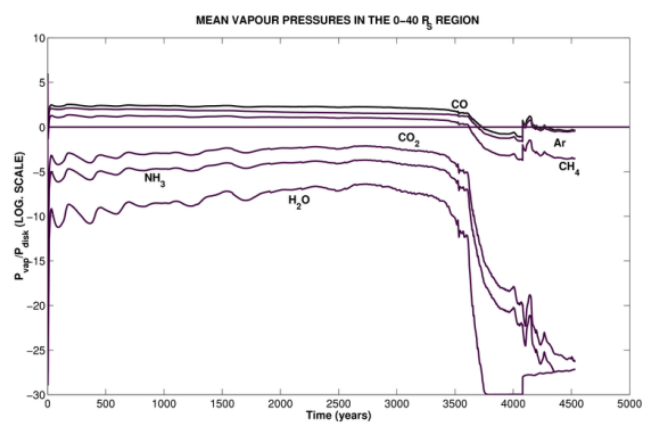

\section{Panel e}

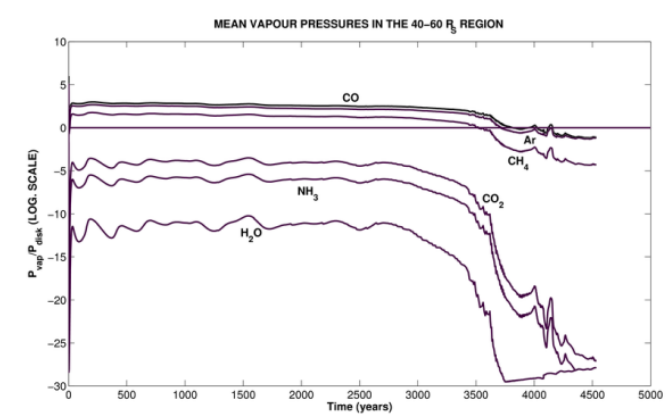




\section{Panel f}

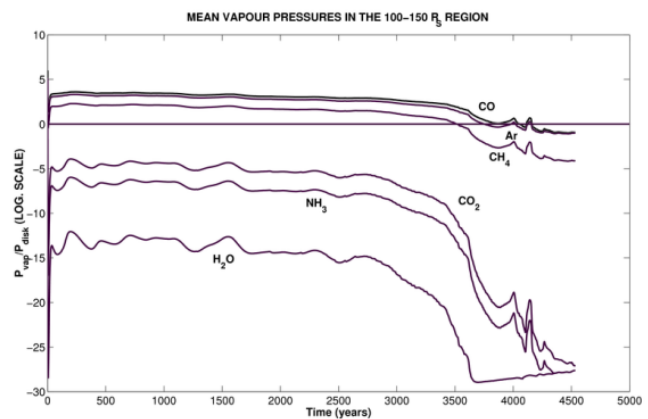

Figure 6: Mean vapor pressures in the Jovian (panels a-c) and the Saturnian (panel d-f) disks as a function of time. Each panel shows the partial vapor pressures of different chemical species $(\mathrm{CO}$, $\mathrm{Ar}, \mathrm{CH}_{4}, \mathrm{CO}_{2}, \mathrm{NH}_{3}, \mathrm{H}_{2} \mathrm{O}$ ) in selected annular shells as those described in Figure 5. The partial vapor pressures are expressed in units of the local gas pressure and in logarithmic scale while the time is expressed in years. 